\title{
La traduction des sonnets de Shakespeare
}

\section{Yves Bonnefoy}

\section{(2) OpenEdition \\ Journals}

\section{Édition électronique}

URL : http://journals.openedition.org/shakespeare/540

DOI : 10.4000/shakespeare.540

ISSN : 2271-6424

Éditeur

Société Française Shakespeare

Édition imprimée

Date de publication : 1 novembre 2000

Pagination : 47-62

ISBN : 2-84269-407-4

\section{Référence électronique}

Yves Bonnefoy, "La traduction des sonnets de Shakespeare », Actes des congrès de la Société française Shakespeare [En ligne], 18| 2000, mis en ligne le 01 novembre 2007, consulté le 01 mai 2019. URL: http://journals.openedition.org/shakespeare/540 ; DOI : 10.4000/shakespeare.540 


\section{S H A K E S P E A R E \\ \& L A F R A N C E}

Société Française Shakespeare

Actes du Congrès de 2000

* * *

Textes réunis et présentés par

Patricia DORVAL

publiés sous la direction de Jean-Marie MAGUIN 
Colloque honoré d'une subvention du Ministère de l'Éducation Nationale, de la Recherche et de la Technologie

Site Internet $:<$ http://alor.univ-montp3.fr/SFS/> Liste de diffusion : <sfs-1@smr1.univ-montp3.fr>

Tous droits de traduction, de reproduction et d'adaptation réservés pour tous les pays.

(C) 2000. Société Française Shakespeare, École Normale Supérieure, 45 rue d'Ulm, 75005 Paris.

ISBN 2-84269-407-4 


\section{LA TRADUCTION DES SONNETS DE S H A K E S PE A R E}

Mesdames, messieurs, vous n'êtes pas de ceux qui se demanderaient s'il y a du sens aujourd'hui à entreprendre encore une fois une traduction des sonnets de Shakespeare.

Par expérience directe d'historiens et de critiques vous savez en effet ce qu'est un texte authentiquement littéraire, et combien il présente d'enchevêtrements et de strates qu'aucune traduction ne peut seule reparcourir ni qu'aucun traducteur n'est évidemment en mesure de savoir tous reconnaître. On peut admettre qu'un roman de faible qualité ne soit traduit qu'une fois sans grand dommage, ce serait déjà plus inquiétant dans le cas d'un essai de quelque importance, car les notions apparemment les plus transparentes ne sont pas sans plis et replis dus à la langue où on les formule, d'où de celle-ci à une autre des écarts dans l'interprétation de la moindre page qu'on n'en finira pas de vouloir analyser, réparer; mais s'il s'agit d'un poème et surtout d'un grand poème, il va de soi qu'aucune traduction n'est capable d'en restituer la richesse ni en droit de prétendre être suffisante et ultime. Les poèmes parlent en nous au plan où nous sommes nous-mêmes des poètes ou à tout le moins cherchons à l'être, ce qui implique qu'à déjà simplement les lire nous sommes engagés dans une écriture qui fait de ce qu'elle perçoit dans leur texte des signifiants dans notre recherche de notre 
rapport à nous-mêmes; ils n'existent donc que dans cet emploi partiellement subjectif, où s'inscrit avec force l'effet de notre situation historique, en perpétuel devenir ; et il est donc naturel que de ces emplois incessants, qui est leur vie même, surgissent à intervalles réguliers des tentatives nouvelles, précipitées même parfois par le changement des époques.

Quant aux sonnets de Shakespeare, il est clair que dans notre langue ce travail, collectif en somme, de redéploiement de leur texte est allé assez loin sur diverses voies, qui partent souvent de leur centre même. Il y a, je le dis d'emblée, de bonnes traductions des sonnets, et même celle de Pierre Leyris, qui est en cours, a toutes les chances d'être remarquable. Avec son autorisation je vais en lire un fragment, le voici (voir ci-après), et je trouverais parfaitement légitime qu'on le préfère à ce que je vous présenterai tout à l'heure, si c'était sur ce plan de la qualité que j'entendais me placer. Mais c'est à tout autre chose que je voudrais vous donner l'occasion de réfléchir.

Cette autre chose, c'est une question de méthode, qui me paraît se poser avec les sonnets de Shakespeare d'une façon qui leur est à la fois particulière et susceptible de généralisation, pour bien d'autres cas.

Je n'ai pas, et je le soulignerai tout d'abord, entrepris de traduire les sonnets comme, par exemple, je l'ai fait pour les poèmes de Yeats, c'est-à-dire motivé simplement par mon souci poétique : lequel, en présence d'une grande œuvre, se serait vu questionné par une intuition, une vision, une écriture également admirables, dont $\mathrm{j}$ 'aurais à prendre mesure dans le seul champ où ce soit possible, à savoir mon propre rapport d'écriture à la poésie. Les sonnets de Shakespeare, c'est aussi de ce point de vue, bien sûr, qu'ils m'ont requis et gardé près d'eux, mais je m'y suis attaché, audelà de quelques premières tentatives, pour deux autres raisons moins directement décidées par cet attachement à leur texte.

La première de ces raisons, c'est une curiosité. J'ai traduit au cours de longues années nombre de pièces de Shakespeare, une douzaine - il est vrai, essentiellement les tragédies -, je les ai donc lues, si je puis dire, lues de cette façon plus approfondie que traduire nous rend possible, puisque au lieu de céder aux 
entraînements de la lecture en surface, voici qu'il nous faut peser chaque mot, retourner le sens de chaque phrase, à l'aide d'éditions critiques qui apportent ce qu'autrement on aurait pu négliger, ou méconnaître, en particulier des significations ou des allusions spécifiques de l'époque élisabéthaine. Et ainsi ai-je pu me faire une certaine idée de Shakespeare où s'inscrivent des caractères de son œuvre qui me semblent très évidents, cette évidence étant soutenue par l'impression d'unité profonde que me donne aussi la quasi totalité de ces pièces. Or, cette idée, je ne la retrouve pas dans les sonnets.

Sans essayer aujourd'hui de développer ce point, je remarquerai, et ce sera donc un simple exemple, qu'il n'y a, à mon sens du moins, aucune misogynie dans le théâtre, ni d'ailleurs non plus dans Vénus et Adonis ou dans Le viol de Lucrèce. Hamlet tient des propos tout à fait insultants à l'égard d'Ophélie et des femmes en général, mais c'est sa mélancolie qui se marque ainsi, et que Shakespeare observe avec le sentiment qu'il y a là une maladie de l'être psychique dont la guérison est ce dont plus tard il fera le sujet du Conte d'hiver. Lady Macbeth ou Goneril sont des figures du mal qui sont des femmes, mais Edmond ou Iago sont tout autant des êtres mauvais, parmi cette fois les hommes. Il est sûr que pour l'auteur de Macbeth, Le Roi Lear et Othello, le mal, dont il fait son souci, n'a pas de sexe. Et dans Antoine et Cléopâtre il y a même une extraordinaire réévaluation de la femme, je m'en suis expliqué dans un essai récent sur - pour reprendre un mot insistant de l'œuvre la «noblesse» de Cléopâtre.

Or, les sonnets... Vous en connaissez évidemment les diatribes à l'égard des femmes, aussi violentes que tristement conventionnelles, et où l'aversion se double de ce qui semble bien une crainte, jamais rencontrée dans les pièces. Je citerai de ce point de vue le sonnet 144 , où le démon est donné pour femme, et la femme pour démon, sans qu'aucune voix de la profondeur du texte ne s'élève pour tempérer, ne serait-ce que d'ironie, cette fureur. Et d'ailleurs s'ajoute à ce sentiment quelque chose encore qui me paraît bien peu shakespearien, la hantise du péché, dont certains sonnets disent l'ampleur étonnante. Péché de l'amour de soi, oui sans doute, dans le sonnet 62 , mais cette obsession de soi est une crainte de l'autre, et péché de luxure, mais parce que cet autre vampirise alors celui qui s'y voue : et cet autre est femme, et cette femme est coupable. Voici ce sonnet 129 , que je cite dans ma traduction pour vous préparer aux remarques que je ferai tout à 
1'heure (voir ci-après).

Le mal, dans les sonnets, est associé à la femme d'une façon tout de même assez nette, autant qu'inquiétante. Le mal, dans le théâtre de Shakespeare, est spontanément et profondément perçu, au contraire, à un plan que je puis dire cosmique, où on le voit transcender ses aspects sociaux ou psychologiques pour se révéler, de façon en vérité mystérieuse, un rapport à soi de l'être parlant, une donnée de l'être fondamentale et que Shakespeare n'hésite pas à affronter comme telle : les quelques tragédies que j'évoquais tout à l'heure n'étant rien d'autre que cette méditation, qui fait la grandeur de l'œuvre. Et vous pressentez sans doute ce que je suis tenté de penser. Oserai-je le dire devant des shakespeariens avertis, et mieux informés que moi ? Je ne doute absolument pas que l'auteur du théâtre soit, pour presque tout dans les pièces, une seule et même personne, que je ne vois aucune raison de dissocier de ce comédien qui est né à Stratford et s'y retira, œuvre faite. Mais on me dirait que l'auteur des sonnets est quelqu'un d'autre, et même socialement tout autre, quelque noble amateur désireux de dissimuler ses poèmes, je n'en serais pas étonné.

J'ai donc commencé de traduire les poèmes pour essayer de mieux pénétrer ce problème ; pour, en somme, me faire une opinion et ainsi vérifier, ou non, ce que je suis tenté de penser de Shakespeare, au seuil d'un travail d'ensemble que j'aimerais bien faire un jour. Mais il y a une autre raison à cette traduction, qui a donc le caractère d'une expérience. Et cette raison, c'est que, quelle que soit la qualité des traductions existantes, je ne suis pas satisfait des partis adoptés par les traducteurs.

\section{III}

Il y a, parmi ces partis, le choix de la prose, dont la vertu est évidemment de laisser libre place aux significations que le traducteur appréhende dans le texte et veut faire entendre. Mais avec cette prose quelque chose d'absolument fondamental est perdu, qui est - en son sens, en ses effets - la décision de Shakespeare de se confier à la forme, aux pouvoirs de la prosodie, au moment où pour lui il s'agissait aussi, si ce n'est d'abord, de penser, d'élaborer une connaissance. Et comme cette décision, suivie d'une invention sur deux voies qui se croisent et recroisent sans cesse, c'est la poésie, par définition, ce qui est perdu dans ces cas, c'est le 
poétique comme tel, avec de proche en proche beaucoup des intuitions que son point de vue permet sur le rapport à soi et au monde. Cette perte, c'est ce que Pierre-Jean Jouve, qui était un grand poète, a bien vu, et il a essayé, traduisant les sonnets en prose, de la réparer en injectant de la forme, si je puis dire, à sa langue très ouvragée. Et je ne dis pas qu'il n'y ait pas dans un tel projet du possible, du grand possible, mais c'est aussi rencontrer le péril d'une certaine préciosité, celle qui naît d'une forme qui peut, en prose, se déployer sans être aussitôt obligée de subir la contestation du puissant courant qui, dans un poème, l'emporterait de vers en vers, l'ouvrant ainsi à une expérience du temps. Cette forme qui reste prose, qui n'est pas interpellée par le temps, par la finitude, peut thésauriser ses trouvailles, autrement dit. Elle est au péril d'une avarice qui essaiera de donner le change par ce qu'on appelle des manières, du maniérisme. Et on peut aimer le maniérisme, mais est-il une suffisante approche quand il s'agit de Shakespeare? Quelles que soient leurs propres affinités avec la sensibilité maniériste, présente sur sol anglais de bien des façons, les sonnets que nous attribuons à Shakespeare restent, résolument, des poèmes.

Puis il y a les traductions en vers, j'entends en vers réguliers, et du point de vue même que je viens de faire valoir, ces traductions peuvent donc prétendre à plus grande et profonde fidélité, elles ont accès à l'expérience de la forme autant qu'à l'intelligence du sens, les données de la poésie sont donc sur leur table. Mais ces traductions-là souffrent, me semble-t-il, de deux maladies chroniques dont il est bien difficile de pallier les tristes effets. Un de ces maux, qu'il m'est déjà arrivé de dénoncer, c'est l'acrobatisme. Il y a un donné de la signification; et, je le crois volontiers, un vrai sentiment chez le traducteur des façons et des pouvoirs de la forme au sein de laquelle cette signification a fleuri et doit à nouveau le faire. Mais il y a un autre donné, et impératif, c'est l'obligation d'en passer pour préserver cette signification, comme évidemment il le faut, par une structure désormais décidée a priori de, disons, dix ou douze pieds, avec peut-être des strophes également décidées d'avance et même, qui sait, des rimes. Et il va s'agir d'ajuster ce qui devient donc un matériau à ce qui devant celui-ci s'annonce comme seulement son futur cadre: ajustement impossible, débordant de par tous ses bords, et qui obligera à des choix dont l'ingéniosité deviendra le principal caractère, aux dépens de l'imprudence et de la générosité innées des décisions 
authentiquement poétiques. Beaucoup du sens aura disparu, qualitativement encore plus que quantitativement. Une mauvaise contrainte mal cachée par de mauvais procédés dans l'écriture engendrera, chez le lecteur, un malaise d'autant plus grand aujourd'hui que l'absence d'unité morale ou métaphysique de notre époque nous a déshabitués de reconnaître dans le vers régulier le reflet d'un consensus à plus haut niveau que simplement une convention littéraire, et de l'aimer pour ce pouvoir de rassemblement, évidemment essentiel.

Dans l'œuvre originale, œuvre de poésie, le sens et la forme naissaient ensemble, ils s'étaient, disons, convaincus l'un l'autre en une avancée tâtonnante parfois, mais qui ne laissait rien au dehors, voilà ce qu'il faut savoir et perpétuer. Et pour pouvoir prétendre à cette préservation, il est donc nécessaire de recourir pour traduire la poésie à un vers libre, celui dont la forme, dégagée des contraintes dues à des conventions arbitraires, naîtrait en nous au cœur et au rythme de notre découverte du sens du texte. Procéder de cette façon faciliterait d'ailleurs cette découverte même, puisque la libre invention formelle est la seule accession possible aux intuitions qui caractérisent la poésie, et ont aidé dans leur temps l'auteur à être poète.

Le vers libre est ainsi le seul parti qui me paraisse acceptable pour la traduction des poèmes, et par exemple pour celle de ces sonnets de Shakespeare. Et comme en ce dernier cas il existe des traductions de cette sorte, en vers libres, vous pourrez peut-être penser que, contrairement à ce que je disais tout à l'heure, je n'ai pas motif à remettre en question l'état présent du problème pour des raisons théoriques.

\section{IV}

Et pourtant je ne me sens pas à mon aise avec les traductions des sonnets que nous avons en vers libres, et cela pour une raison qui n'a certes pas trait à leur qualité comme telle, mais à des décisions qui consciemment ou non furent prises à leur début par les traducteurs, et qu'il me paraît utile d'expliciter, de problématiser et d'offrir à la réflexion.

D'un mot, et pour l'essentiel: ce qui me gène dans ces traductions, c'est qu'elles sont juxtalinéaires.

Juxtalinéaires, on voit bien pour quelles raisons elles le sont, et 
peuvent même être décidées les seules possibles quand il s'agit d'un sonnet, que celui-ci soit écrit en anglais ou dans d'autres langues. Traduire vers par vers, en effet, avec, à la rigueur des enjambements ou rétroactions minimes qui rentrent vite dans le rang et ne compromettent pas la survie de la strophe comme la veut le poème, c'est la seule façon pour qu'à la fin il y ait en français quatorze vers comme c'est déjà le cas dans ce texte, d'une façon qui est assurément essentielle et devrait donc être préservée. Essentielle, cette structure du sonnet, parce que, d'une part, elle fonctionne avec une particulière efficace, succession, en nombre de cas, de deux strophes paires et de deux strophes impaires, ce qui assure au point de partage un passage que je dirai, en bref, celui de l'espace au temps, de l'intemporel au devenir, des essences à l'existence. Mais essentielle aussi dans le champ des significations historiques, toujours si actives dans l'écriture, puisque les sonnets ont été à des époques bien définies un aspect fondamental de l'invention collective, avec de ce fait inscription, dans la substance de chacun d'eux, de grands systèmes de la pensée et de la sensibilité qu'ils ont aidés par leur forme même à mûrir, ainsi le néoplatonisme, lui aussi articulation des essences et de l'existence. Inutile de vous rappeler tout ce qui se joue dans le pétrarquisme, et qu'on retrouve accumulé dans les sonnets précisément parce que ce sont des sonnets, parce que ce sont des structures de quatorze vers distribués en quatre strophes.

Je comprends donc bien qu'on ressente le besoin de préserver cette grande et belle et signifiante structure, mais l'obligation qu'on se fait d'être juxtalinéaire ne peut assurément qu'accroître le risque d'acrobatisme et de perdre avec lui ce qu'on aura gagné à sauver le nombre quatorze, cela et bien plus encore. Et c'est en ce point que je m'intéresse à traduire les sonnets de Shakespeare, tout ou partie, parce que je crois qu'une autre façon de faire est concevable, qui elle permettrait la pleine liberté du vers libre tout en conservant l'essentiel du parti prosodique qu'est le sonnet.

Cette façon, cette méthode? C'est de considérer que pour préserver la forme propre au sonnet shakespearien, il n'est pas nécessaire de se limiter à quatorze vers. Ce sonnet de Shakespeare, remarquons-le tout d'abord, ne se présente pas en deux strophes de quatre vers suivies de deux autres de trois, comme il en va des sonnets en France, par exemple chez Du Bellay. Et de ce fait la référence platonicienne, si elle a sens pour Shakespeare, n'est pas perceptible en tout cas dans la distribution des quatorze vers en huit 
et six, - il y a là, soit dit au passage, matière à réflexion sur les voies du métaphysique dans la conscience élisabéthaine. Nous avons à entendre trois strophes de longueur égale et une plus brève, qui de ce fait affichent autre chose que le sonnet français, donnant plutôt à méditer dans les deux derniers vers une pointe, une formulation ramassée et frappante. Et c'est là pencher vers le procédé rhétorique plutôt qu'en rester dans l'expérience de poésie, c'est là poser autrement le rapport du poétique et du rhétorique.

Mais c'est là aussi faciliter le problème du traducteur. Car il apparaît moins nécessaire de bâtir la traduction sur l'opposition du pair et de l'impair, trois strophes longues et une courte peuvent suffire à refléter la structure du sonnet shakespearien, et pourquoi faudrait-il alors que ces strophes «longues», par exemple, aient nécessairement dans la traduction quatre vers? Trois strophes de quatre ou cinq ou six vers suivies d'une de deux ou trois, cela ne donnerait-il pas plus ou moins la même impression que la forme du texte anglais, impression qui semble se concentrer sur le raccourci final avec son effet de surprise ?

Je consens que quelque chose est perdu : par exemple, à se donner ainsi du champ dans la concision de tel ou tel vers, une netteté du parti, une qualité du jeu d'ombres et de lumières dont la précision de découpe consonne avec l'articulation savante des figures au sein de la signification. Mais qu'est cette perte auprès de celle du contrepoint que les sonorités si subtiles de ces vers de Shakespeare apportaient à leur sens, elles qui sont irrémédiablement perdues avec les mots anglais qui les portent ? Et en revanche ne doit-on pas constater que si une strophe peut devenir un ensemble de vers plus long, cela va permettre à l'idée qui y est active de se développer dans la traduction de façon plus naturelle, sans être meurtrie par des artifices, et, en somme, de respirer : la concision, les condensations, les ellipses pouvant alors naître dans la parole du traducteur comme ce que son libre regard a su retrouver dans le texte d'origine, et non comme le produit de ses propres contraintes, lui bien étranger au sens de l'œuvre. Un ou deux vers de plus, s'ils sont nés comme forme qui va, qui cherche, qui reste ainsi au plan de la poésie, et l'idée incluse dans le poème peut mieux fleurir.

Et on va dire que c'est peut-être là un inconvénient tout autant qu'un avantage, car c'est donc préserver beaucoup de cette idée, avec ses figures, ses raisonnements, ses images, alors qu'aura disparu, je le disais à l'instant, la musique originale du texte, qui faisait corps avec elle. Une musique, une vraie traduction en a une. 
Mais à naître d'autant plus aisément que le vers libre l'y aide, ne va-t-elle pas faire négliger de ressentir ce que le son original avait de nécessaire à l'écoute de ce sens même que l'on a su maintenir ? Je suis sensible à cette objection, sachant bien ce que la lecture à voix haute d'un sonnet de Shakespeare par un Anglais peut apporter, dans la densité des strophes, de richesse allitérative, de timbres, de joie sonore associable même à ce qu'a parfois de triste le sens, alors que nos assonances à nous, hautement possibles dans ces vers libres, et capables d'aller fort loin dans l'élaboration musicale, n'obéiront tout de même plus au même rythme tendu qu'assurait la forme plus stricte. Dans la traduction non juxtalinéaire le sens, autrement dit, serait comme porté en avant, avec ce risque aussi, sérieux au plan théorique, qu'on pourrait oublier qu'à chaque instant sens et forme avaient eu même pas et même poids dans l'unité inhérente à l'acte d'écrire.

Et c'est là une objection qui m'empêcherait d'adopter la méthode dont je parle s'il s'agissait pour moi de traduire un sonnet de Sidney, par exemple. Je ne chercherais pas une autre méthode, d'ailleurs, je renoncerai à l'entreprise. Il va de soi aussi que je ne voudrais rien «déployer» d'un vers de Hamlet ou du Roi Lear, qui sont pris dans l'urgence d'une action au cœur de laquelle le sens est donné et reçu d'un coup, antérieurement à toute analyse. J'ai critiqué une fois un projet de traduction «développée» de Hamlet et je ne suis pas en train de changer d'avis.

Mais quelque chose des sonnets de Shakespeare me paraît justifier, au moins dans une certaine mesure, de prendre le risque de donner à l'idée en eux un surcroît de présence dans la traduction qu'on en fait. Et c'est ce que je crois pouvoir appeler leur aspect rhétorique, autrement dit la présence en eux, la plupart des fois, d'une argumentation, comme si c'était un avocat qui parlait, qui y plaidait une cause, démontrant au passage la véracité d'une proposition, d'un précepte. Pensez à ces sonnets du début du livre, qui tentent de convaincre le «beau jeune homme» qu'il a le devoir de procréer, de rebâtir au profit du monde la beauté qui va se défaire en lui. Le discours y est évident, ses enchaînements, leur logique, sont essentiels à ces textes, aucune richesse de la musique des mots ne peut vraiment dès lors se soustraire à notre impression qu'elle est 
aussi, ou parfois d'abord, la vêture de ce plaideur qui reprend la parole au sonnet 3 , disons, après le sonnet 2 , et ensuite. Et de ce point de vue il faut bien penser que la strophe libre, celle qui a loisir de faire place à tout le raisonnement, c'est tout de même cela seul qui permet d'entendre suffisamment en français ce qui est dans le texte anglais sa raison d'être, au moins apparente.

En somme, la méthode de traduction que j'ai essayée, ce qui me paraît la justifier, c'est qu'il y a dans les sonnets de Shakespeare un rapport aussi spécifique que primordial entre le poétique, comme nous l'entendons aujourd'hui, et le rhétorique, si ce n'est pas l'oratoire. Rapport dont je ne dis nullement, faut-il le souligner, qu'il ait lieu aux dépens de la poésie - de l'invention, de la connaissance, spécifiquement poétiques -, car, d'évidence, le poétique foisonne à d'autres plans de cette écriture, par exemple dans les modulations, elles-mêmes de la musique, que l'idée subit en son avancée, au centre parfois perceptible et parfois non de son cortège d'images. Modulations, entre véhémence et doute, entre évidence et énigme, que justement la strophe moins resserrée permettra aussi de mieux faire entendre.

Je vais maintenant vous lire quelques-unes de ces traductions qui peuvent avoir seize ou dix-sept vers, la quatrième strophe restant de toute façon plus courte que les trois autres. 


\section{SONNET CXIIV}

J'ai deux amours, l'un fait ma joie, l'autre m'accable, Tels deux génies qui m'assaillent sans cesse.

Le bon, un ange, est homme, et la beauté même,

Le mauvais, un démon, est femme, de couleur laide.

Pour me jeter plus vite en enfer, mon fléau,

La femme, incite mon bon ange à m'abandonner.

Elle veut corrompre mon saint ami, en faire un diable, Elle veut séduire

Cet être pur, de toute son infâme lubricité.

Et si mon ange est devenu démon, Je peux le soupçonner, non l'affirmer, Toutefois, ils sont loin de moi tous deux, ils sont amis, Et je crois donc que l'ange est en enfer.

Mais je ne puis en avoir le fin mot Et vivrai dans le doute, jusqu'au jour

Où le mauvais démon aura chassé l'ange. 


\section{SONNET CXXIX}

La luxure : naufrage, en abîme de honte, De la force vitale. Rien qu'en pensée Elle est parjure, meurtrière, elle répand Coupablement le sang, elle est sauvage, Excessive, brutale et cruelle, traîtresse,

Et méprisée si tôt que satisfaite, Follement poursuivie mais follement Haïe, le hameçon qu'on a dans la bouche Fait pour que l'esprit sombre, par la douleur.

Et insensée à vouloir comme à prendre, Rage de qui a eu, qui possède, qui cherche, Désirée, un délice, éprouvée, un malheur, Attendue, une joie, passée, l'ombre d'un songe,

Et cela, qui ne le sait pas ? Mais qui sait se garder De ce ciel qui voue l'homme à cet enfer ? 


\section{SONNET XVIII}

Vais-je te comparer à un jour d'été ?

Mais tu as plus de charme et plus de douceur,

Car les bourgeons de mai, si tendres, d'âpres vents

Les malmènent encore,

Et l'été est un bail de durée trop brève.

Et trop d'ardeur a parfois l'œil du ciel

Ou trop souvent c'est son or qui se voile,

Et beauté se défait en beauté même

Par accident, ou ce change qui est la loi du monde.

Tandis que toi, ton éternel été

$\mathrm{Ne}$ se fanera pas. Il n'abdiquera pas

Sa beauté, ta richesse. Dans ses ténèbres

La mort ne pourra pas se vanter que tu erres:

Tant il est vrai qu'en ces rets éternels,

Mes vers, tu ne feras que croître, avec le temps.

Aussi longtemps qu'hommes respireront, Aussi longtemps que des yeux sauront voir, Vivront ces vers, qui te donneront vie. 


\section{SONNET LV}

Ni le marbre, ni la lumière d'or des monuments

Que les Princes érigent, ne vivront plus

Que ce puissant poème ; où tu resplendiras

De plus d'éclat que ces pierres qu'insultent

Les marques noires du temps qui souille tout.

La guerre dévastatrice renversera

Les statues; à grand bruit elle arrachera

Les racines des murs ; mais ni l'épée de Mars

Ni la furie des flammes du pillage

Ne détruiront ces vers où vivra ta mémoire.

Contre la mort, contre 1'oubli hostile

Tu marcheras! Ton éloge aura sens

Même au regard des âges qui fibre à fibre Déferont l'univers, y mettront fin.

Avant qu'au Jugement tu ne te redresses

Tu vivras dans mes vers,

Tu étincelleras dans ces yeux d'un amant. 


\section{SONNET LXXIII}

Contemple en moi ce moment de l'année

Où les feuilles des arbres ont jauni, Puis sont tombées; et peu pendent encore

À leurs branches qui s'ébouriffent dans le froid :

Chapelles nues en ruines,

Où les chantres, ce furent tard des chants d'oiseaux.

Contemple en moi la journée qui s'achève, La trace à l'Occident que le soleil laisse Mais que bientôt les ombres de la nuit, Cette autre mort, effaceront, qui cousent Pour le repos les paupières de tout.

Contemple en moi le rougeoiement d'un feu Qui gît parmi les cendres de sa jeunesse, Ce lit de mort sur lequel il lui faut Expirer, de par l'ardeur même qui l'a nourri.

Contemple, et contempler fasse ton amour Plus fort, d'avoir aimé, d'avoir su aimer Ce que dans peu de temps il te faudra perdre. 


\section{SONNET 71}

NO LONGER MOURN FOR ME WHEN I AM DEAD...

Traduction de Pierre Leyris

Garde-toi, quand je serai mort, de mener deuil Plus longtemps que ne sonne le glas monotone Grâce auquel le monde apprendra que j'ai fui, Ce vil monde, au profit des vers plus vils encore.

Oui, si tu viens alors à lire ce poème, Oublie, oublie la main qui l'a tracé : je t'aime Au point que je me veux banni de tes pensers Si pour y prendre place, je dois t'attrister.

Oui, si tu viens jamais à relire ces vers Quand je serai, disons, à l'argile mêlé, Ne prononce pas seulement mon pauvre nom, Mais laisse ton amour s'éteindre avec ma vie.

De crainte que les gens avisés ne se rient De ta peine pour t'en mieux faire moquerie. 


\section{SONNET LXXI}

Ne me pleure pas plus longtemps, quand je serai mort,

Que ne retentira le sombre glas

Qui mornement annoncera au monde

Que j'en ai fui la vilénie, pour vivre

Parmi les vers, certes plus vils encore.

Et même, si tu lis ces lignes, n'aies aucun

Souvenir de la main qui les a tracées,

Car si songer à moi dût te faire souffrir,

Je t'aime tellement que je préfère

$\mathrm{Ne}$ pas rester dans tes tendres pensées.

Oui, je dis bien, si tu lis ce poème,

Quand peut-être déjà je ne serai qu'argile,

$\mathrm{Ne}$ te redis pas même mon pauvre nom,

Laisse ton affection finir avec ma vie ;

J'ai trop peur que le monde trop raisonnable

N'épie le deuil que te serait ma mort, Et ne m'emploie pour se railler de toi. 